\title{
AN IMPROVED DECOMPOSITION APPROACH AND ITS COMPUTATIONAL TECHNIQUE FOR ANALYZING PRIMAL-DUAL RELATIONSHIP IN LP \& LFP PROBLEMS
}

\author{
H. K. Das ${ }^{1}$ and M. Babul Hasan ${ }^{2}$ \\ ${ }^{l}$ Department of Mathematics and Statistics, Concordia University, \\ Montr'eal, Quebec, H3G 1M8 Canada \\ ${ }^{2}$ Department of Mathematics, University of Dhaka, Dhaka-1000, Bangladesh \\ E-mail: ${ }^{1}$ hkdas.rohit@gmail.com, ${ }^{2}$ mbabulhasan@yahoo.com
}

Received 04-11-2012 Accepted 30-10-2013

\begin{abstract}
In this paper, we study the methodology of primal dual solutions in Linear Programming (LP) \& Linear Fractional Programming (LFP) problems. A comparative study is also made on different duals of LP \& LFP. We then develop an improved decomposition approach for showing the relationship of primal and dual approach of LP \& LFP problems by giving algorithm. Numerical examples are given to demonstrate our method. A computer programming code is also developed for showing primal and dual decomposition approach of LP \& LFP with proper instructions using AMPL. Finally, we have drawn a conclusion stating the privilege of our method of computation.
\end{abstract}

Keywords: LP, LFP, Primal-Dual, Decomposition and Computer Algebra

\section{Introduction}

In 1960's and 1970's several authors, Swarup [17], Chadha [5], Kaska[12], Gol'estein [9,10], Sharma and Swarup [16], Seshan [16] and Joshi, Singh and Gupta[11] and many other authors proposed different types of methods for dual problems related to the primal LFP problems consisting of optimizing linear fractional objective function subject to a system of linear constraints. Most of the authors proposed a dual form in which the objective function is linear. Some of them are based on the well-known Charnes and Coopers transformation [4] leads to the duality theory of LP. Only Sharma and Swarup [16] and Seshan [17] have defined a dual form in which the objective function is fractional, that is, ratio of two linear functions. All these duals proposed by different authors are actually equivalent to one another. Most of the authors proved all the duality theorems and did not apply the decomposition primal dual approach to solve LFP problems. Walker [18], Kornbluth and Salkin [13] stated the decomposition approach for LFP. Also Dantzig \& Wolfe [7], Dantzig [6], Wolfe [19], Baumol and Fabian [1] are silent about the LFP decomposition but in this paper we show their LP decomposition is sophisticated not only for solving LFP but also in primal dual approach of LFP. Every 
mathematical programming problem has an associated dual problem. For any primal problem, a dual problem can be constructed which is very closely connected with the original problem. In this paper, we study the methodology of optimal dual solution in LP \& LFP and improve a decomposition approach to furnish the complete primal dual solutions which depends on DWD. We also develop a computational technique for showing optimal dual decomposition approach of LP \& LFP using programming language AMPL [8].

The rest of the paper is organized as follows. Section 2 is based on the discussion of primal dual LP \& LFP problems. Section 3 and 4 are based on the formulation and algorithms of Kornbluth \& Salkin and our improved optimal dual decompose of LP \& LFP problems respectively. In Section 5, we demonstrate of our improved decomposed algorithm by a numerical example. In Section 6, we develop a computer programming code using the programming language AMPL. In section 7, we present the comparison of different methods.

\section{Discussions of Primal Dual LP \& LFP}

In this section, we briefly discuss about the primal dual LP \& LFP model and its complexity in the real world problems. We also precisely discuss about the formulation of LP \& LFP problems.

\subsection{Primal Dual Approach of Linear Programming (LP) [18]}

The linear decomposition, the final tableaux of the master program and subprograms provide not only the primal solution but also the full dual evaluation. Consider the following LP problem

$$
\begin{gathered}
\text { primal Maximize } c_{1} \lambda_{1}+c_{2} \lambda_{2}, \quad \text { subject to: } A_{1} \lambda_{1} \leq b_{1}, \quad A_{2} \lambda_{2} \leq b_{2} \\
\qquad \begin{array}{c}
M_{1} \lambda_{1}+ \\
M_{2} \lambda_{2} \leq b \\
\lambda_{i} \geq 0
\end{array}
\end{gathered}
$$

Here, the equation (*) is called complex constraints and $M_{1}, M_{2}$ are the coefficients of the equation $(*)$.The dual solutions of this primal are given as follows.

$$
\begin{aligned}
& \text { Minimize } \pi_{\alpha} b_{\alpha}=\pi_{1} b_{1}+\pi_{2} b_{2}+\pi_{b} b \\
& \text { subject to: } \pi_{\alpha} A_{\alpha}+\pi_{b} M_{\alpha} \geq c_{\alpha}, \quad \pi_{\alpha} \geq 0, \alpha=1,2
\end{aligned}
$$

The dual variables $\pi^{*}{ }_{\alpha}$ for (i) and (ii) according as $\alpha=1,2$. Walker's[18] proof relies on the linear duality theorems equality the optimal primal and dual objective functions. So it is clear that: optimal objective function=dual objective functions i.e. $c_{1} \lambda_{1}^{*}+c_{2} \lambda_{2}^{*}=\pi_{1} b_{1}+\pi_{2} b_{2}+\pi_{b} b$

\subsection{Primal Dual Approach of Linear Fractional Programming (LFP) [13]}

The LFP decomposition problem involving two divisions or sub problems can be defined as in the following ways: 


$$
\begin{gathered}
\text { Maximize : } f=\frac{c_{1} \lambda_{1}+c_{2} \lambda_{2}}{d_{1} \lambda_{1}+d_{2} \lambda_{2}+\beta} \\
\text { subject to: } \quad A_{1} \lambda_{1} \leq b_{1} \\
A_{2} \lambda_{2} \leq b_{2} \\
M_{1} \lambda_{1}+M_{2} \lambda_{2} \leq b \\
\lambda_{i} \geq 0
\end{gathered}
$$

Here, the equation (iii) is called complex constraints and $M_{1}, M_{2}$ are the coefficients of the complex constraints. Hence $\pi_{1}, \pi_{2}$ and $\pi_{b}$ are the duals of (i), (ii) and (iii) respectively. Hence primarily,

$$
\frac{c_{1} \lambda^{*}{ }_{1}+c_{2} \lambda^{*}{ }_{2}}{d_{1} \lambda^{*}{ }_{1}+d_{2} \lambda^{*}{ }_{2}+\beta} \neq \pi^{*}{ }_{1} b_{1}+\pi^{*}{ }_{2} b_{3}+\pi_{b}^{*} b
$$

Hence $\lambda^{*}{ }_{1}, \lambda^{*}{ }_{2}$ are primal solutions and $\pi^{*}{ }_{1}, \pi^{*}{ }_{2}$ and $\pi^{*}{ }_{b}$ are the dual solutions.

\section{Formulation of Kornbluth \& Salkin Optimal Dual Solution [13]}

In this section, we present the Kornbluth \& Salkin's optimal dual solution in LFP problems. A dual problem to (3) is find $\theta=\left(\pi_{1}{ }^{*}, \pi_{2}{ }^{*}, \pi^{*}, \lambda^{*}{ }_{1}, \lambda^{*}{ }_{2}\right)$ where $\theta$ being the solution to the dual problem.

$$
\begin{aligned}
& \text { dual. } \pi_{1} b_{1}+\pi_{2} b_{2}+\pi_{b} b \\
& \text { subjectto: } \pi_{1} A_{1}+\pi_{b} M_{1} \geq\left[\frac{\partial f}{\partial \lambda_{1}}\right]_{\lambda=\lambda^{*}}, \pi_{2} A_{2}+\pi_{b} M_{2} \geq\left[\frac{\partial f}{\partial \lambda_{2}}\right]_{\lambda=\lambda^{*}}, \pi_{1}, \pi_{2}, \pi_{b} \geq 0
\end{aligned}
$$

Hence for $\lambda_{1}^{*}, \lambda_{2}^{*}$ and $C_{\alpha i}^{-}=\left[\frac{\partial f}{\partial \lambda_{i}}\right]_{\lambda=\lambda^{*}}$

where $\lambda^{*}$ is optimal for (3) and from the primal (3) we get the new LP in equation (7).

\begin{tabular}{|cc|cc|}
\hline Maximize $\bar{c} c_{1} x_{1}+\bar{c}_{2} x_{2}$ & & Maximize $\sum \mu_{1}^{i} \bar{c}_{1} \lambda_{1}^{i}+\mu_{2}^{j} \overline{c_{2}} \lambda_{2}^{i}$ & \\
subjectto: $\quad A_{1} x_{1} \leq b_{1}$ & $(i)$ & subjectto: $\sum \mu_{1}^{i} M_{1} \lambda_{1}^{i}+\mu_{2}^{j} M_{2} \lambda_{2}^{j} \leq b$ & $\left(i^{\prime}\right)$ \\
$A_{2} x_{2} \leq b_{2}$ & $(i i)$ & $\sum \mu_{1}^{i}=1$ & $\left(i^{\prime} i^{\prime}\right)$ \\
$M_{1} x_{1}+M_{2} x_{2} \leq b, x_{i} \geq 0$ & $($ iii $)$ & $\sum \mu_{2}^{j}=1$ & $\left(i^{\prime} i^{\prime} i^{\prime}\right)$ \\
\end{tabular}

Then (7) is a linear decomposition problem. At this point sub-program for (7) are given as follows. 


$$
\begin{aligned}
& \text { Maximize } \sum_{i}\left(\bar{C}_{\alpha i}-\hat{\pi} . M_{\alpha_{i}}\right) \lambda_{i}, \quad \text { subjectto: } \quad A_{\alpha 1} \lambda_{\alpha} \leq b_{\alpha}, \quad \lambda_{\alpha} \geq 0 \quad \alpha=1,2 \\
& \text { Minimize } \pi_{\alpha} b_{\alpha} \text {, subject to } \pi_{\alpha} A_{\alpha} \geq \bar{C}_{\alpha}-\hat{\pi} \cdot M_{\alpha_{i}} \\
& \pi_{\alpha} \geq 0 \quad \alpha=1,2
\end{aligned}
$$

The dual to these two sub-problems are as follows.

\subsection{Kornbluth \& Salkin Optimal Dual Decomposition Algorithm [13]}

In this section, we present the Kornbluth \& Salkin optimal dual decompose algorithm for LFP.

Step (a): Assume $k_{\alpha}$ solutions from division $\alpha=1,2$.

Step $(b)$ : From the master problem (M) and optimize using, say, the CC method. Let, $x=x_{1}, x_{2}$ be the 'optimal' program derived.

Step $(c)$ : Derive the marginal values of production for $x_{1_{i}}, x_{2_{i}}$ at the present solution

lavels; i.e., the vector $\left(\xi_{1}, \xi_{2}\right)$ whose components are $\xi_{\alpha i}=\left[C_{\alpha i}-\pi . M_{\alpha_{i}}\right]$ where $\left[C_{\alpha i}^{-}=\frac{\partial f}{\partial \lambda_{i}}\right]_{\lambda=\alpha} \alpha=1,2$

Step $(d)$ : Present each division with these new marginal figures and request optimization of each of the sub-problems with respect to the new (linear) objective: $\max \xi_{\alpha} \cdot x_{\alpha}=f_{\alpha}$ subject to $A_{\alpha} \cdot x_{\alpha} \leq b_{\alpha}$ and $x_{\alpha} \geq 0, \alpha=1,2$.

Step(e): Test for optimality with the new division solutions. Let, $\bar{f}_{\alpha}$ be the optimal value of $f_{\alpha}$; then, if $\sigma_{\alpha} \geq \bar{f}_{\alpha}(\alpha=1,2)$ the present solution is optimal, but, if $\sigma_{\alpha} \leq \bar{f}_{\alpha}(\alpha=1,2)$ update $k_{\alpha}$ and go to Step (b). The details are discussed in [14].

Since primal dual LP \& LFP relation is strongly visible. So our aim is to show the effectiveness. In the next section, we present an improve decomposition approach for optimal dual LP \& LFP.

\section{Formulation of Our Improved Primal Dual Solution}

In this section, we present our improved decomposition technique. For this, the original LFP problem make into LP using (6). The whole process will be carried out by the following mathematical formulations. Let, the original LP problem be 


$$
\begin{array}{cc}
\text { Maximize: } & \bar{c}_{1} x_{1}+\bar{c}_{2} x_{2} \\
\text { subject to: } & A_{1} x_{1} \leq b_{1} \\
A_{2} x_{2} \leq b_{2} \\
M_{1} x_{1}+M_{2} x_{2} \leq b \\
x_{i} \geq 0
\end{array}
$$

The improved decomposition principles composed of the following sub problems with the help of Lagrangian relaxation.

\begin{tabular}{|l|c|}
\hline $\begin{array}{l}\text { Sub-problem } \\
\text { primal }\end{array}$ & $\begin{array}{l}\text { Master problem } \\
- \\
- \\
\text { Maximize: }: c_{1} x_{1}+c_{2} x_{2}-\lambda_{k}\left(M_{1} x_{1}+M_{2} x_{2}-b\right)\end{array}$ \\
subjectto: $: A_{1} x_{1} \leq b_{1}, \quad A_{2} x_{2} \leq b_{2}, \quad x_{i} \geq 0 \quad$ (13) & subjectto : $\left(M_{1} x_{1}+M_{2} x_{2}\right) \eta_{k} \leq b \quad$ (14) \\
& $\eta_{k}=1, \eta_{k} \geq 0$ \\
\hline
\end{tabular}

Iteration number $K$ is continuously increased until the optimality condition is satisfied.

\section{Optimality condition}

The value of the sub-problem will be equal to the value of the master problem i.e. $V(S)=$ $V(M)$.

\section{Getting the primal solution}

The master problem $M(\eta)$ contains the final solution in $\eta$ variables. To convert the solution to the $x$ variables, we have to calculate the optimal solution $x^{*}$ by the formula $x^{*}=\sum_{l=1}^{k} \eta_{l} x_{i}^{l}$.

\subsection{Improved Primal Dual Decomposition Algorithm}

In this section, we briefly discuss our improved optimal dual decompose algorithm for solving LP \& LFP problems.

Step (a): Check that problem is LP or LFP. If LP go to Step (e) Otherwise go to Step (b).

Step (b): Assume $k_{\alpha}$ solutions from division $\alpha=1,2$.

Step (c): From the master problem (M) and optimize using, say, the Charces \& Copper method. Let, $x=x_{1}, x_{2}$ be the 'optimal' program derived. 
Step (d): Derive the new marginal values $\left[C_{\alpha i}^{-}=\frac{\partial f}{\partial \lambda_{i}}\right]_{\lambda=\alpha} \alpha=1,2$ for creating LP (12) at the present solution labels $x_{1_{i}}, x_{2_{i}}$ from LFP (3).

Step (e): Decompose the LP problem (12) into sub problem (13) and master problem (14). First, solve the sub problem using arbitrary dual $\lambda_{1}$. Then follow the following Steps.

Step $(f)$ : Using sub problem value solves the master problem.

Step $(g)$ : From master problem, we are able to obtain dual prices for each of the constraints.

Step $(h)$ : This information is then utilized in the objective function of the sub problem.

Step $(i)$ : The sub problem is solved.

Step $(j)$ : Check that $V(S)=V(M)$. If the optimality condition fails go to the following Step.

Step $(k)$ : A new variable is then added to the master problem and the master problem is re-solved. Re-solving the master problem will generate new dual values. Then go to the Step (h) and the process is repeated until the optimality condition is satisfied.

\section{Numerical Experimental Results}

In this section, we present a numerical example to demonstrate of and our improve decomposition technique to show the optimal dual algorithm of LP \& LFP Problems.

Numerical Example: 5.1 This numerical example is taken from Kornbluth \& Salkin[13].

$$
\begin{gathered}
\text { Maximize } \frac{4 x_{1}+2 x_{2}+4 y_{1}+3 y_{2}}{x_{1}+x_{2}+y_{1}+y_{2}+12.5} \\
\text { subject to: } \quad x_{1}+2 x_{2}+2 y_{1}+y_{2} \leq 15, \quad x_{1}+3 x_{2} \leq 30,2 x_{1}+x_{2} \leq 20 \\
\quad y_{1} \leq 10, y_{2} \leq 10, y_{1}+y_{2} \leq 20, \quad x_{i} \geq 0, y_{i} \geq 0
\end{gathered}
$$

Solution: The optimal solution which can be found by any method such as Charnes and Copper (CC) method is $f^{*}=2, x_{1}=10, x_{2}=0, y_{1}=2.5, y_{2}=0$.

The dual variables are $\pi_{b}=\frac{1}{25}, \pi_{1}=\left(0, \frac{1}{25}\right), \pi_{2}=(0,0,0)$ For the first set of decompose we have tendered the first basic plans $\underset{-}{x}=\left(\begin{array}{l}10 \\ 0\end{array}\right),\left(\begin{array}{l}0 \\ 0\end{array}\right)$, the second basic

$$
\begin{array}{ll}
\text { plans } & \begin{array}{l}
y=\left(\begin{array}{l}
10 \\
0
\end{array}\right),\left(\begin{array}{l}
0 \\
0
\end{array}\right) \\
c_{11}=\frac{\partial f}{\partial x_{1}}=\frac{\left(x_{1}+x_{2}+y_{1}+y_{2}+12.5\right) \cdot 4-\left(4 x_{1}+2 x_{2}+4 y_{1}+3 y_{2}\right) .1}{\left(x_{1}+x_{2}+y_{1}+y_{2}+12.5\right)^{2}} c_{12}=0
\end{array}
\end{array}
$$


$c_{21}^{-}=\frac{2}{25} c_{22}^{-}=\frac{1}{25}$ Now the converted linear fractional programming to linear programming objective function is given as

Maximize $\bar{c}_{1} x_{1}+\bar{c}_{2} x_{2}=\bar{c}_{11} x_{1}+\bar{c}_{12} x_{2}+\bar{c}_{11} y_{1}+\bar{c}_{12} y_{2}=\frac{2}{25} x_{1}+\frac{2}{25} y_{1}+\frac{1}{25} y_{2}$

subject to: $x_{1}+2 x_{2}+2 y_{1}+y_{2} \leq 15, x_{1}+3 x_{2} \leq 30,2 x_{1}+x_{2} \leq 20$

$$
y_{1} \leq 10, y_{2} \leq 10, y_{1}+y_{2} \leq 20, x_{i}, y_{i} \geq 0 \text {. }
$$

\subsection{Solution using Kornbluth \& Salkin}

In this section, we present a numerical example to demonstrate Kornbluth \& Salkin decomposition technique. This is clearly shown in the reference.

Kornluth \& Salkin decomposition method, terminates if $\sigma_{\alpha} \geq f_{\alpha}$ otherwise go to step (b). Since the $1^{\text {st }}$ division dual $\sigma_{1}=\frac{10}{25}$ and optimal $\hat{f}_{1}=\frac{10}{25}$ and satisfies $\sigma_{1}=f_{1}$.

Also the $2^{\text {nd }}$ division dual $\sigma_{2}=0$ and optimal $\hat{f}_{1}=0$ and satisfies $\sigma_{2}=f_{2}$. So this solution is optimal. Applying the algorithm in Section 3.1, we get optimal\& dual solution from $(15)$ is $\sigma_{b}=\frac{1}{25}, \pi_{1}^{*}=(10,0), \pi_{2}^{*}=(0,0,0)$.

\subsection{Solution Using Our Improved Decomposition Method}

In this section, we present an example to demonstrate our improved decomposition technique.

\section{Sub-problem for $k-t h$ iteration}

We apply the Lagrangian relaxation by relaxing constraint (*), we get the general subproblem and the last iteration is given in the following way.

Maximize $\frac{2}{25} x_{1}+\frac{2}{25} y_{1}+\frac{1}{25} y_{2}-\lambda_{k}\left(x_{1}+2 x_{2}+2 y_{1}+y_{2}-15\right)$

subject to $\quad x_{1}+3 x_{2} \leq 30,2 x_{1}+x_{2} \leq 20, y_{1} \leq 10, y_{2} \leq 10, y_{1}+y_{2} \leq 20, x_{i}, y_{i} \geq 0$

\section{Master problem for $k-t h$ iteration}

Maximize $\quad \sum_{i=1}^{k} \eta_{i}\left(\frac{2}{25} x^{k}+\frac{2}{25} y^{k}{ }_{1}+\frac{1}{25} y^{k}{ }_{2}-15\right)$

subject to

$$
\sum_{i=1}^{k} \eta_{i}\left(x_{1}^{k}+2 x_{2}^{k}+2 y_{1}^{k}+y_{2}^{k} \leq 15, \quad \sum_{i=1}^{k} \eta_{i}=1, \quad \eta_{i} \geq 0\right.
$$




\section{Iteration-4 (For k=4): Sub-problem}

$$
\begin{array}{ll}
\text { Maximize } & \frac{2}{25} x_{1}+\frac{2}{25} y_{1}+\frac{1}{25} y_{2}-\frac{1}{25}\left(x_{1}+2 x_{2}+2 y_{1}+y_{2}-15\right) \\
\text { subject to } & x_{1}+3 x_{2} \leq 30,2 x_{1}+x_{2} \leq 20, \quad y_{1} \leq 10, y_{2} \leq 10, y_{1}+y_{2} \leq 20, x_{i}, y_{i} \geq 0
\end{array}
$$

Solving by simplex method we get $x_{1}=10, x_{2}=0, y_{1}=\frac{5}{2}, y_{2}=0$ and sub-problem values $z=S_{4}(v)=1$.Also the dual solution is $\sigma_{b}=\frac{1}{25}=\lambda_{3}, \pi_{1}^{*}=(10,0)$, $\pi_{2}^{*}=(0,0,0)$.

\section{Master problem}

Maximize $\quad 0 . \eta_{1}+\frac{9}{5} \eta_{2}+\frac{20}{25} \cdot \eta_{3}+\frac{20}{25} \cdot \eta_{4}$

$$
\text { subject to: } \quad \text { 0. } \eta_{1}+35 \eta_{2}+10 . \eta_{3}+10 . \eta_{4} \leq 15, \quad \eta_{1}+\eta_{2}+\eta_{3}+\eta_{4}=1, \quad \eta_{i} \geq 0
$$

Solving by Dual Simplex Method we get $\eta_{1}=0, \eta_{2}=0, \eta_{3}=0, \eta_{4}=1$ and master problem value $z=M_{4}(v)=1$.The first dual value $\lambda_{4}=\frac{1}{25}$ and the second dual value is $\frac{10}{25}$. Since $S_{4}(v)=1=M_{4}(v)$.

Thus the current solution is optimal with the solution $x_{i}=\sum_{k=1}^{K} x(k, i) \eta(1, k)$, $(i=1,4)$

$x_{1}=0 \cdot 0+10 \cdot 0+10 \cdot 0+10 \cdot 1=10, x_{2}=0 \cdot 0+0 \cdot 0+0 \cdot 0+0 \cdot 1=0$

$y_{1}=0 \cdot 0+0 \cdot 0+10 \cdot 0+\frac{5}{2} \cdot 1=\frac{5}{2}, y_{2}=0 \cdot 0+0 \cdot 0+5 \cdot 0+0 \cdot 1=0$

which gives $x_{1}=10, x_{2}=0, y_{1}=\frac{5}{2}, y_{2}=0$ with maximum of $z=1$.

On the other hand the dual objective function value is $15 \cdot \frac{1}{25}+\frac{10}{25}=1$.

Solving the total problem using Charnes and Cooper method or Kornluth \& Salkin decomposition method, one can observe that our primal \& dual objective function values will coincide with their results.

\section{Programming Code in AMPL}

In this section, we developed a computational technique corresponding to our improved decomposition technique for primal dual LP and LFP using the programming language AMPL.

\section{Result \& Discussion}

In this section, we present the solution comparison between manual output and the programming output. Then we present the convergence of the master problem \& Sub problem. 


\subsection{Solution Comparison}

In the current section, the values we calculated manually earlier and the values we have now from our programming output are tabulated.

Table1: Comparison of solutions

\begin{tabular}{|c|c|c|}
\hline $\begin{array}{c}\text { Iterati } \\
\text { on }\end{array}$ & Manual Output & Program Output \\
\hline 1 & 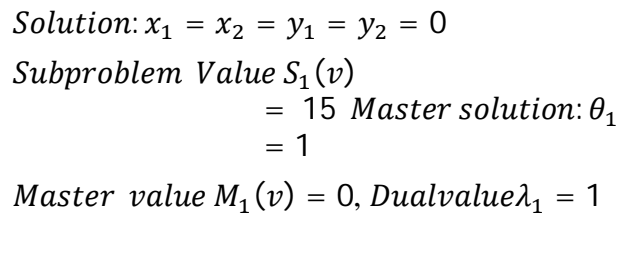 & $\begin{array}{l}\text { Solution: } x_{1}=x_{2}=y_{1}=y_{2}=0 \\
\text { Subproblem value } S_{1}(v)=15 \\
\text { Master solution: } \theta_{1}=1 \\
\text { Master value } M_{1}(v)=0 \\
\text { Dual value } \lambda_{1}=1\end{array}$ \\
\hline 2 & $\begin{array}{l}\text { Solution: } x_{1}=10, x_{2}=0, y_{1}=10, y_{2}=5 \\
\text { and sub-problem value } z=S_{2}(v)= \\
\frac{9}{5} . \text { Master problem solution: } \\
\quad \eta_{1}=4 / 7, \eta_{2}=3 / 7 \text { and master value } \\
z=M_{1}(v)=27 / 35 \text { and dual } \lambda_{2}=0\end{array}$ & $\begin{array}{l}\text { Solution: } x_{1}=10, x_{2}=0, y_{1}=10, y_{2}=10 \\
\text { and sub-problem value } z=S_{2}(v)=2 \\
\text { Master problem solution: } \\
\qquad \eta_{1}=0.625, \eta_{2}=0.375 \text { and master } \\
\text { problem value } z=M_{1}(v)=0.75 \\
\text { the dual value } \lambda_{2}=0\end{array}$ \\
\hline 3 & $\begin{array}{l}\text { Solution: } \quad x_{1}=10, x_{2}=0, y_{1}=0, y_{2}=0 \\
\text { and sub-problem value } z=S_{2}(v)=\frac{37}{35} \\
\text { Master: } \\
\qquad \eta_{1}=0, \eta_{2}=1 / 5, \eta_{3}=4 / 5 \\
\quad \text { And master value } z=M_{1}(v)=1 \\
\text { Dual value } \lambda_{3}=9 / 175\end{array}$ & $\begin{array}{l}\text { Solution: } x_{1}=10, x_{2}=0, y_{1}=0, y_{2}=0 \\
\text { and sub-problem value } z=S_{2}(v)=1.05 \text {. } \\
\text { Master: } \\
\qquad \eta_{1}=0, \eta_{2}=1 / 5, \eta_{3}=4 / 5 \\
\quad \text { and master value } z=M_{1}(v)=1, \\
\text { Dual value } \lambda_{3}=1 / 25\end{array}$ \\
\hline 4 & $\begin{array}{l}\text { Solution: } x_{1}=10, x_{2}=0, y_{1}=\frac{5}{2}, y_{2}=0 \text { and } \\
\text { Subproblem } S_{4}(v)=1 \text {. } \\
\text { Master problem solution: } \\
\qquad \eta_{1}=0, \eta_{2}=0, \eta_{3}=0, \eta_{4}=1 \\
\text { master value } z=M_{4}(v)=1 \text { and } \\
\text { dual value } \lambda_{4}=1 / 25\end{array}$ & $\begin{array}{l}\quad \text { Solution: } x_{1}=10, x_{2}=0, y_{1}=0 \\
y_{2}=0 \text { and subproblem value } z=S_{4}(v)= \\
\text { 1. Master Solution: } \\
\qquad \eta_{1}=0, \eta_{2}=0.166666, \\
\eta_{3}=0.8333333, \eta_{4}=0 \\
\text { master value } z=M_{4}(v)=1 \text { and } \\
\text { dual value } \lambda_{4}=1 / 25 .\end{array}$ \\
\hline
\end{tabular}

Form the Table 1, we can say that our computer technique gives the identical optimal value of the objective function and the optimal solutions. Since the original problem has alternative solution so it gives the alternative solutions. 


\subsection{Convergence of Master and Sub Problem Value}

In this section, we present the graphical representation of the convergence of master and sub problem values using manual and coding results. We have used few commands in MATHEMATICA which gives the following figure.

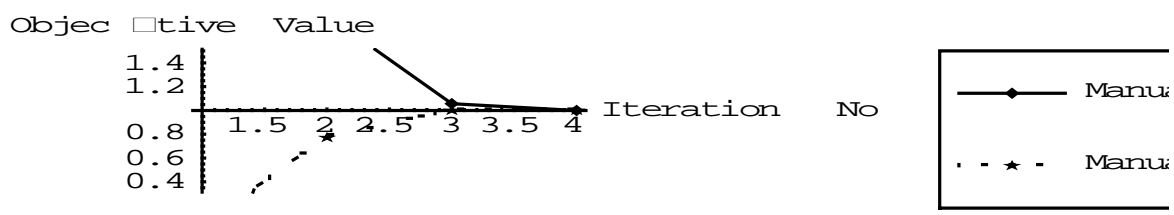

Fig. 1 Convergence of Manual Sub-problem and Master-problem value of Example 5.1

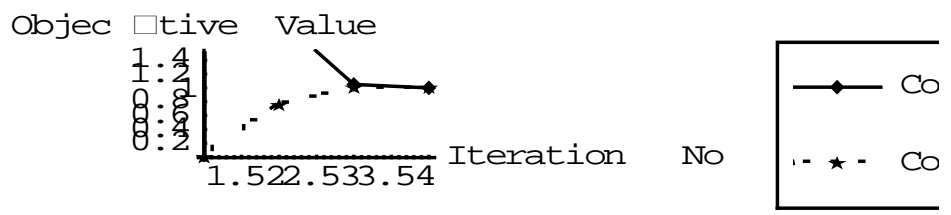

Fig. 2: Convergence of Coding Sub-problem and Master-problem value of Example 5.1

\section{Conclusion}

In this paper, we developed a sophisticated decomposition algorithm for showing the relationship of primal and dual approach of LP \& LFP which was demonstrated with a numerical example. A computational technique is also developed using AMPL. A comparative study is also given between Kornbluth \& Salkin and our improve decomposition LFP method. We conclude that our technique is more efficient for using primal dual LP \& LFP problems than the others.

\section{REFERENCE}

[1] Baumol W.J., T. Fabian, "Decomposition Pricing for Decentralization and External Economies," Management Sciences, 11,1964, pp.1-32.

[2] Bector, C.R., "Duality in Fractional and Indefinite programming", 48(6), 1968.

[3] Bector, C.R., "Duality in Linear Fractional programming", Utilitas Mathematica, 4,1973, pp.155-168.

[4] Charnes, A and Cooper, W.W., "Programming with Linear Fractional Functionals", Naval Research Logistics Quarterly, 9(3), 1962, PP. 181-186. 
[5] Chadha, S.S and Chadha, Veena, "Linear Fractional programming and Duality", 2007 pp. 119-125. (C) Springer-Verlag.

[6] Das H. K. \& M. Babul Hasan, " A Proposed Technique for Solving Linear Fractional Bounded Variable Problems", The Dhaka University Journal of Science, 60(2), 2012, 223230.

[7] Dantzig, G. B. and P. Wolfe, "The Decomposition Algorithm for linear Programming", Econometrica, 29(4), 1961.

[8] Fourer, R., D.M. Gay and B.W. Kernighan, "A Modeling Language for Mathematical Programming", Second edition, Thomson Publication, 2003.

[9] Gol'stein, E.G., "Dual Problems of Convex and Fractional Convex Programming in Functional Spaces", Doklady Academic Nauk SSSR, 172(5),1967, pp.1007-1010.

[10] Gol'stein, E.G., "Duality Theory in Mathematical Programming and its Applications", Nauka, Moscow, 1971.

[11] Joshi, V. D., Singh, E., Gupta, N., "Primal Dual Approach to Solve Linear Fractional Programming Problem", J. of Applied Mathematics, Statistics and Informatics, 4(1), 2008.

[12] Kaska, J, "Duality in Linear Fractional Programming", Ekonomicko - Matematicky Objor, 5(4), 1969, pp. $442-453$.

[13] KornbluthJ. S. H., G. R. Salkin,"The Optimal Dual Solution in Linear Fractional Decomposition Problems", Operation Research. 22(1),1974, pp-183-189.

[14] KornbluthJ. S. H, Aspects of Mathematical Programming in Financial Corporate Planning, Ph.D. Thesis, University of London, 1970.

[15] Seshan, C.R., "On Duality in Linear Fractional Programming", Proc. Indian Acad. Sci., Sect. A. Math. Sci., 89, 1980 pp. $35-42$.

[16] Sharma, I.C, and Swarup, K, "On Duality in Linear Fractional Programming", Zeitschrift fur Operations Research, Band 16, Seite $91-100$.

[17] Swarup, K., "Duality in Fractional Programming", Unternehmensforschung, Band 12, Heft 2, 1968, pp. 106-112.

[18] Walker. E.,"A Method for the Optimal Dual Solution to a Linear Decomposition,"Operation Research, 17, 1969, pp.368-370.

[19] Wolfe, P., "The simplex method for quadratic programming",Econometrica, 27(3), 1959, pp.382-398. 